\title{
Introduction to Artificial Intelligence and the Media
}

\section{Taina Pihlajarinne and Anette Alén-Savikko}

\section{EVOLUTION AND REVOLUTION IN THE AI ERA}

The media industry has always been changing in close connection to technological development, whether in terms of the printing press or radio waves. Currently, artificial intelligence (AI) and automation are key drivers in the (r)evolution of the ways in which news and other media content is produced, distributed, and consumed. Increased use of AI and automation in media practices and the underlying technical architecture implies changes to journalistic work and challenges conventional media roles and functions. Nonetheless, the public's right to information should be preserved alongside civil rights, while the idea of journalism still includes (maybe more than ever) expectations of accountability: its task is to serve and answer to the public, to act responsibly. ${ }^{1}$

New technologies are expected to increase efficiency in the newsroom: many routine tasks are apt for automation; consumption patterns become highly measurable, while AI-based content production gains new ground. For their part, online platforms for content-sharing and social networking moderate and curate their catalogue and newsfeed with the help of AI-based solutions. As its 'fuel', the use of AI and automation relies on massive amounts of data, and utilisation of data is becoming an integral part of media practices. This is not without legal and policy implications: First, AI and automation rely on data utilisation. This in turn means reassessing data ownership and intellectual property (IP) rights. Second, AI and algorithmic control are accompanied by issues involving agency and responsibilities, whether in terms of authorship,

1 Robert G Picard and Victor Pickard, 'Essential Principles for Contemporary Media and Communications Policymaking' (2017) Reuters Institute for the Study of Journalism 7, 30-31 http://reutersinstitute.politics.ox.ac.uk/publication/essential -principlescontemporary-media-and-communications-policymaking accessed 23 January 2020. 
public interest rationales, or private law liability. These aspects raise fundamental questions about how rights and responsibilities should be reconsidered in the light of incentives for accountable utilisation of AI.

Utilisation of AI affects the media sector in a wide sense, from legacy media to online platforms, including social media sites. Moreover, the changes go deep into the institutional fabric of the media. It is increasingly important to view the media sector from a holistic perspective instead of as separate silos. ${ }^{2}$ It is no longer clear-cut where to draw the lines of editorial conduct, while challenges arise both for conventional approaches regarding the role and functions of the media as well as in terms of incentives and conditions for the media to operate in the digital environment and in today's data-driven society. ${ }^{3} \mathrm{AI}$ and algorithms also pose challenges for editorial decision-making and content production by introducing 'outsiders' to the newsroom and to journalistic practices, such as programmers and developers. At the same time, the public is being exposed to new types of threats and forms of opacity, especially in terms of privacy and algorithmic decision-making. Personal data is increasingly used by the media in order to monitor consumption and customise production, while AI-based solutions are adopted to facilitate content management.

These developments take us back to fundamental questions, such as: what is 'journalism' or 'news'. They also imply a need to revisit basic rationales and justifications of media regulation as well as familiar demands for accountability. Reliance on data makes a case for examining data protection and IP laws. Moreover, use of AI highlights questions of agency, entitlements, and obligations, especially as the human contribution becomes increasingly detached from automated (autonomous) processes and end products. From a legal perspective, this translates into questions of authorship and (among others) private law liability. This edited volume examines the feasibility of principles and concepts as currently applied in the light of incentivising accountability and puts forward alternative solutions. Accountable use of AI reduces negative societal impacts, including those related to harms to privacy and the credibility of journalism as well as overprotection of IP rights, while promoting positive societal impacts, such as efficient and high-quality content production.

Andrew Chadwick, The Hybrid Media System: Politics and Power (OUP 2013). Philip Michael Napoli, Audience Evolution: New Technologies and the Transformation of Media Audiences (Columbia University Press 2011); Chris Peters and Marcel Broersma (eds), Rethinking Journalism Again: Societal Role and Public Relevance of in a Digital Age (Routledge 2016); Philip Michael Napoli (ed), Mediated Communication. Handbooks of Communication Science (Mouton De Gruyter 2018). 


\section{REGULATION, RIGHTS AND RESPONSIBILITIES}

The legal and policy dimensions are of fundamental importance for the future of the media and journalism. Importantly, investing in and relying on new technologies in the media sector requires some degree of predictability, while policy-makers are in desperate need of evidence-based solutions. At the same time, however, it has become clear that the concept of AI needs to be explained and anchored in journalistic practices so as to enable informed policy and law-making. For their part, regulatory and self-regulatory tools may work as incentives for accountability. Then again, the market for AI-based solutions is developing even as we speak. The role of global online platforms has been questioned not only by legacy media companies but also by legislators. However, these platforms have considerable power in terms of developing media practices and constructing their own rights and responsibilities, for instance in the field of copyright or data protection. ${ }^{4}$ There is clearly a need for a more principled approach that draws from ethics and shared societal values. ${ }^{5}$ However, conventional principles are seldom applicable as such in the context of AI and automation, but rather require in-depth analysis. Moreover, in many cases, the legislative framework is in a state of flux and lagging behind real-life development. This is true in the case of automated or AI-based content supervision and user data processing by online platforms. Indeed, many policy and legislative solutions have rather operated on a piecemeal basis or have seemed rather uncritically to be drawing from conventional regulatory models and following a one-sided economic logic. ${ }^{6}$

During the finishing stages of this volume, the EU Commission released its draft framework for regulating AI. On 21 April 2021, the Proposal for a Regulation laying down harmonised rules on artificial intelligence (Artificial Intelligence Act $)^{7}$ was introduced. Due to the timing of this book, most of the

4 Jockum Hildén, The Politics of Datafication: The Influence of Lobbyists on the EU's Data Protection reform and its Consequences for the Legitimacy of the General Data Protection Regulation (University of Helsinki 2019) http://urn.fi/URN:ISBN:978 -951-51-3410-3 accessed 24 June 2021.

5 Picard and Pickard (n 1) 5-10.

6 See also Andrej Savin, 'Regulating Internet Platforms in the EU - The Emergence of the "Level Playing Field" (2018) 34(6) Computer Law \& Security Review 1215-1231 https://doi.org/10.1016/j.clsr.2018.08.008.

7 Commission, 'Proposal for a Regulation of the European Parliament and of the Council laying down rules on artificial intelligence' (Artificial Intelligence Act) and amending certain Union legislative acts COM (2021) 206 final (21 April 2021) https://eur-lex.europa.eu/legal-content/EN/ALL/?uri=CELEX:52021PC0206 accessed 16 May 2021. 
authors have not been able to take account of the proposal in their chapters. However, the approaches adopted and connections between this volume and the proposal are briefly touched on here, in Mark Leiser's opening chapter as well as in the Conclusions. The proposal for an Artificial Intelligence Act aims to safeguard a functioning internal market as far as AI systems are concerned, at the same time stressing fundamental rights, trustworthiness, and ethics. The proposal essentially relies on a risk-based approach and aims to establish harmonised rules in the European Union. ${ }^{8}$ The proposal identifies and acknowledges both the societal benefits and the new types of risks involved in using AI. ${ }^{9}$ This 'duality' is closely similar to the approach of this volume, which tackles both rights and responsibilities in automated media. As far as regulatory tools are concerned, similarly to the core of this volume, the proposal for an Artificial Intelligence Act in the EU claims to offer a 'balanced regulatory approach' ${ }^{10}$

\section{STRUCTURE AND CONTENTS OF THE VOLUME}

This volume discusses pressing issues at the intersections of AI, the media, and the law under an umbrella of critically re-examining rights and responsibilities from the perspectives of incentives for accountable utilisation of AI. The volume asks: (1) What do 'AI' and 'accountability' mean in terms of media practices, principles, and power relations? (2) How to address the (r)evolution with informed law and policy in order to incentivise accountable utilisation of AI and reduce negative societal impacts? What kind of solution offers a balanced outcome? Both legislative and ethical solutions related to these themes are analysed. With regard to the Proposal for the Artificial Intelligence Act, this volume may be read both as complementary and as a source of critique. Complementarity takes place especially where the specifics of AI in the media sector are concerned, while critique mainly relates to the more principled or rights-oriented approach that this volume is seeking.

In Part I on 'Journalistic Principles and Artificial Intelligence', the focus is on general legal and policy issues at the intersections of AI and journalism from the perspective of incentives for accountable utilisation of AI in the media. In setting the scene, Leiser discusses journalistic endeavours and the risks of AI, especially from the perspective of some inherent biases that are involved in the utilisation of AI and automation. Leiser sheds light on some inaccuracies and discriminatory features that training data contains or contrib-

Ibid., 1-3, 7, 12-14.

Ibid., 1-3.

Ibid., 3. 
utes to. In doing so, he also ends up questioning assumptions of objectivity traditionally linked to journalism, data, and AI. Nonetheless, the importance of 'exposure diversity' 11 is acknowledged in the context of AI solutions - a topic of utmost importance in a world filled with disinformation of various sorts.

Alén-Savikko elaborates on the concept of transparency in data and computational journalism, analysing it in terms of both law and ethics. She explores potential 'rights' to identify computer-generated content and to access information about the background data and logics of algorithmic news production. However, transparency proves to be a rather peculiar demand and one which is somewhat difficult to turn into actual practice. These questions go beyond what transparency is as a data protection issue. However, since personal data is involved in the processes around AI systems, it is essential to discuss relevant aspects of European data protection law in the context of automated media.

Korpisaari elaborates on the 'journalistic exemption' in the EU General Data Protection Regulation (GDPR). This also leads to interesting questions about journalism itself.

Part II of this volume, dedicated to 'Trust, Disinformation and Platforms', deals with the role, rights, and obligations of platforms as well as the power relations involved in their operation from the perspective of incentives for accountable utilisation of AI. For their part, Napoli and Graf offer an approach to social media platforms as public trustees. By this, they mean a model familiar in the US broadcasting sector. Napoli and Graf ask whether it could apply to social media so as to offer solutions to the challenges involved in disinformation.

Tong addresses practices of policing content on social media platforms such as Facebook and Twitter by addressing three dilemmas: she addresses trust, freedom of information, and liability and their implications for ethics and law.

Social media recommender systems and their commercial unfairness are addressed by Goanta and Spanakis. As a result of the increase in social media users earning a living with content creation, the underlying power imbalance becomes evident - while lack of transparency in algorithmic operations is apt to make matters worse. This calls for approaches that draw from unfair competition.

In Part III, the topic of 'Remits and Limits of Exclusive Rights' is discussed from the perspective of intellectual property (IP) law. The chapters look at the guiding principles, concepts, and new types of rights in the field of IP law, mainly copyright, as well as limitations to those rights. Exclusive rights are examined from the standpoint of incentivising content production powered by

11 See also Natali Helberger and Mira Burri, 'Public Service Media and Exposure Diversity’ (2015) 9 International Journal of Communication 1319-1323. 
AI and taking into account societal impacts, such as those related to freedom of information. Part III addresses essential questions related to the incentive function of copyright. This is examined critically in the light of AI-based content production, while the need to rethink legal personality is discussed in the context of creative processes. Thereby, pressing questions of appropriate forms of stimuli and legal status with regard to AI are addressed in the context of the media in particular. Moreover, access to data is discussed as a crucial issue with regard to use of AI in the media sector.

For his part, Rognstad addresses AI and European copyright law in terms of works having to be the results of authors' own intellectual efforts. He contributes to efforts to rethink the current legislative framework in order to make room for AI in content production.

Ballardini and van den Hoven van Genderen explore the question of AI as a legal subject in the specific context of automated media.

For their part, Pihlajarinne, Thesleff, Leppänen, and Valmari analyse some specific problems relating to AI-based journalism in terms of incentives, current copyright doctrines, and problems of overly extensive or mistargeted IP protection.

Vesala discusses the newly introduced EU press publishers' right from the perspective of AI, while particularly targeting the effects of the new right on news production and services powered by AI.

Finally, Ørstavik takes a look at access to data for training of algorithms. She discusses the prospects of IP protection, while joining in the wider policy debate on the role of IP rights as mechanisms of stimuli and control.

\section{ABOUT THE TOPIC}

The writing of this volume took place in the middle of the global Covid-19 pandemic. If nothing more or less, we have seen not only the importance of the media in our society, but also witnessed the power of recommender systems, coordinated campaigns involving disinformation and many other topics addressed in this volume. The following chapters - although originally not intended by the authors - will now be read through the lens of the glasses that this global experience has placed on our noses. 